PROCEEDINGS OF THE

AMERICAN MATHEMATICAL SOCIETY

Volume 138, Number 2, February 2010, Pages 417-425

S 0002-9939(09)10136-3

Article electronically published on October 1, 2009

\title{
LIE SUPERAUTOMORPHISMS ON ASSOCIATIVE ALGEBRAS
}

\author{
YURI BAHTURIN AND MATEJ BREŠAR \\ (Communicated by Gail R. Letzter)
}

\begin{abstract}
The results on Lie homomorphisms of associative algebras are extended to certain associative superalgebras. It is shown that under appropriate conditions a Lie superautomorphism of $A=A_{0} \oplus A_{1}$ is a sum of a superautomorphism or the negative of a superantiautomorphism and a central map. In particular we consider the situation when $A$ is a central simple algebra and its $\mathbb{Z}_{2}$-grading is induced by an idempotent.
\end{abstract}

\section{INTRODUCTION}

Let $A=A_{0} \oplus A_{1}$ be an associative superalgebra. Then $A$ becomes a Lie superalgebra if we replace the associative product by the superbracket $[a, b]_{s}$. The relationship between the associative and Lie structure of $A$ was studied by many authors; see for example [3, 4, 7, 10, 11, 13, 15, 16, 18. However, to the best of our knowledge, the natural problem of finding the precise connection between the homomorphisms of these two structures did not receive due attention in the literature. The purpose of this paper is to begin filling this gap.

In the classical ungraded case, the results on Lie homomorphisms of associative rings and algebras have also been obtained with some "delay" if compared to other Lie structure results. These latter had already been obtained in the 1950s and 1960s by Herstein and some of his students; see 12. Martindale solved various Lie map problems somewhat later (see e.g. [14]), however, under an assumption that the rings in question contain nontrivial idempotents. The first result avoiding idempotents was obtained in 1993 by the second author [8. The methods from 8 and related papers were later extended in various directions, and eventually this resulted in the creation of the theory of functional identities; see [9]. Among various applications of functional identities, solutions of several Lie map problems are particularly notable. Functional identities will be used, indirectly but essentially, also in this paper.

We shall say that a bijective linear map $\varphi: A \rightarrow A$ is a Lie superautomorphism of $A$ if $\varphi\left(A_{i}\right)=A_{i}, i=0,1$, and $\varphi\left([a, b]_{s}\right)=[\varphi(a), \varphi(b)]_{s}$ for all $a, b \in A_{0} \cup A_{1}$. We will show that under certain favorable conditions $\varphi$ can be expressed through superautomorphisms or superantiautomorphisms and central maps. The main result (Theorem 3.1) describes some abstract conditions, which are then applied to the

Received by the editors March 30, 2009.

2000 Mathematics Subject Classification. Primary 17B40; Secondary 16W10, 16R50, 17 B60.

The first author was partially supported by NSERC grant \#227060-04 and URP grant, Memorial University of Newfoundland.

The second author was partially supported by ARRS grant \#P1-0288. 
case when $A$ is a central simple algebra and the natural $\mathbb{Z}_{2}$-grading is induced by an idempotent (Corollary 3.2). The main idea of the proof is to introduce a usual Lie automorphism of the Grassmann envelope of $A$ and then apply the theory from 9. Here we were influenced by our recent work [1, 2, where we noticed that some results from [9] are applicable to the tensor products of commutative algebras with rather general algebras. In a certain sense, Grassman algebras are very close to commutative algebras and Grassman envelopes are very close to tensor products. This was a good motivation for the present work.

We remark that an analogous concept of a Jordan superhomomorphism was treated in [6], but using a more straightforward and elementary approach.

We do not try to push the results to their utmost generality in this short paper. Our main goal is to present the method which, as we hope, could be extended to more general contexts. We plan to continue the investigation of Lie superhomomorphisms in a more technical work in the near future.

After preparing a draft of this paper, we received a preprint of Wang [17] in which Lie superhomomorphisms have also been considered. While there is some overlap between his paper and ours, there are also essential differences. Wang does not reduce the problem to the usual Lie maps in associative algebras (as we do, using the Grassmann envelope), but studies functional identities directly in associative superalgebras. Also, he imposes the conditions on the odd part $A_{1}$, while our restrictions concern the even part $A_{0}$.

\section{Preliminaries}

By "an algebra" we shall always mean an algebra over a fixed field $F$ with $\operatorname{char}(F) \neq 2$. Mostly we will consider associative algebras, but not exclusively. So the term "algebra" can mean a nonassociative algebra. For convenience we assume that all our associative algebras have an identity element.

2.1. Superalgebras. Recall that a superalgebra is a $\mathbb{Z}_{2}$-graded algebra $A=A_{0} \oplus$ $A_{1}, A_{i} A_{j} \subseteq A_{i+j}$ where $i, j \in \mathbb{Z}_{2}$. Elements from $A_{i}$ are said to be homogeneous of degree $i, i=0,1$. For $x \in A_{i}$ we shall write $|x|=i$.

An important example of a superalgebra is the Grassman superalgebra, $G$. As an algebra, $G$ is just an associative algebra generated by elements $1, e_{1}, e_{2}, \ldots$ that satisfy $e_{i}^{2}=e_{i} e_{j}+e_{j} e_{i}=0$ for all $i, j$; as a superalgebra, it is determined by the following rule: $1 \in G_{0}, e_{i_{1}} e_{i_{2}} \ldots e_{i_{k}} \in G_{0}$ if $k$ is even and $e_{i_{1}} e_{i_{2}} \ldots e_{i_{k}} \in G_{1}$ if $k$ is odd. Now let $A=A_{0} \oplus A_{1}$ be an arbitrary superalgebra. The algebra $G(A)=G_{0} \otimes A_{0}+G_{1} \otimes A_{1}$, which we view as a subalgebra of the tensor product $A \otimes G$, is called the Grassman envelope of $A$. If $G(A)$ is a Lie algebra, then we say that $A$ is a Lie superalgebra. Similar definitions make sense for other varieties of algebras. In particular, if $G(A)$ is an associative algebra, then we say that $A$ is an associative superalgebra. But actually it is easy to see that an associative superalgebra is nothing but a $\mathbb{Z}_{2}$-graded associative algebra. On the other hand, a Lie superalgebra is not a Lie algebra if its grading is nontrivial. Lie superalgebras can be equivalently defined through the super-anticommutativity of the product and the super-Jacobi identity. But we shall not need them in this paper.

Let $A=A_{0} \oplus A_{1}$ be an associative superalgebra. The superbracket of two homogeneous elements $a, b \in A$ is defined as $[a, b]_{s}=a b-(-1)^{|a||b|} b a$. We extend $[., .]_{s}$ by bilinearity to $A \times A$. Then $A$, endowed with the superbracket together with the original grading and the original vector space structure, becomes a Lie 
superalgebra. The supercenter of $A$ is defined as the set of all $a \in A$ such that $[a, A]_{s}=0$. Note that a Lie superautomorphism $\varphi: A \rightarrow A$ satisfies $\varphi\left(\left[a_{0}, b\right]\right)=$ $\left[\varphi\left(a_{0}\right), \varphi(b)\right]$ for all $a_{0} \in A_{0}, b \in A$, and $\varphi\left(a_{1} \circ b_{1}\right)=\varphi\left(a_{1}\right) \circ \varphi\left(b_{1}\right)$ for all $a_{1}, b_{1} \in A_{1}$. Here, of course, $[u, v]=u v-v u$ and $u \circ v=u v+v u$.

Let $A$ be an associative algebra and $e$ an idempotent in $A$. If we set

$$
A_{0}=e A e+(1-e) A(1-e) \quad \text { and } \quad A_{1}=e A(1-e)+(1-e) A e,
$$

then $A$ becomes an associative superalgebra. This is the basic example of a superalgebra structure on an associative algebra, and often this is, in fact, the only example possible. Indeed, let $A=A_{0} \oplus A_{1}$ be an arbitrary associative superalgebra. Then $\sigma\left(a_{0}+a_{1}\right)=a_{0}-a_{1}$ defines an automorphism of $A$ such that $\sigma^{2}=$ id. If $\sigma$ is inner, then there exists an invertible $u \in A$ such that $u^{2}$ lies in the center of $A$ and $A_{0}=\{x \in A \mid[u, x]=0\}, A_{1}=\{x \in A \mid u \circ x=0\}$. Assume further that $u^{2}$ can be written as a square of some central element, $u^{2}=c^{2}$. Then we may replace $u$ by $c^{-1} u$ and therefore assume without loss of generality that $u^{2}=1$. Hence $e=\frac{1}{2}(1-u)$ is an idempotent, and one can easily show that $A_{0}$ and $A_{1}$ are given as in (11). Thus, for instance, if an associative algebra $A$ is such that it has only inner automorphisms, its center is just $F$, and $F$ is an algebraically closed field, then every superalgebra structure of $A$ arises from an idempotent.

The prototype example of (11) is $M(p \mid q)$, the algebra of square matrices of order $p+q$ equipped with the following $\mathbb{Z}_{2}$-grading: $M(p \mid q)_{0}$ consists of matrices of the form $\left[\begin{array}{cc}A & 0 \\ 0 & D\end{array}\right], A \in M_{p}(F), D \in M_{q}(F)$, and $M(p \mid q)_{1}$ consists of matrices of the form $\left[\begin{array}{ll}0 & B \\ C & 0\end{array}\right], B \in M_{p, q}(F), C \in M_{q, p}(F)$.

2.2. The strong degree. The concept of the strong degree was introduced in [5] and is also exposed in [9, Chapter 2]. We will now give a very brief survey which is sufficient for our purposes.

Let $A$ be an associative algebra. By $M(A)$ we denote the multiplication algebra of $A$, that is, the algebra of linear operators on $A$ generated by all left and all right multiplications $L_{a}$ and $R_{b}, a, b \in A$. Thus a typical element in $M(A)$ is an operator on $A$ of the form $x \mapsto \sum_{i=1}^{n} a_{i} x b_{i}, a_{i}, b_{i} \in A$.

Let $t \in A$ be a nonzero element, and let $n \geq 0$ be an integer. We say that the strong degree of $t$ is greater than $n, s$-deg $(t)>n$, if for every $0 \leq i \leq n$ there exists $\mathcal{E}_{i} \in M(A)$ such that $\mathcal{E}_{i}\left(t^{j}\right)=\delta_{i j}$ for each $j=0,1, \ldots, n$ (here $\delta_{i j}$ is the "Kronecker delta", and $\left.t^{0}=1\right)$. Clearly, in this case, $1, t, \ldots, t^{n}$ are linearly independent. If $s-\operatorname{deg}(t)>n-1$ but $s-\operatorname{deg}(t) \ngtr n$, then we say that the strong degree of $t$ is $n$ $(s-\operatorname{deg}(t)=n)$. If $s-\operatorname{deg}(t)>n$ for every positive integer $n$, then $s-\operatorname{deg}(t)=\infty$. Finally, the strong degree of $A$ is $s-\operatorname{deg}(A)=\sup \{s-\operatorname{deg}(t) \mid t \in A\}$. Trivially, $s-\operatorname{deg}(A) \geq 1$ for every algebra $A$.

Let us record three simple lemmas. The first two can be very easily checked, and we omit the proofs.

Lemma 2.1. If $A^{\prime}$ is a subalgebra of $A$ such that $A^{\prime}$ contains the identity element of $A$, then $s-\operatorname{deg}(A) \geq s-\operatorname{deg}\left(A^{\prime}\right)$.

Lemma 2.2. If $A_{1}$ and $A_{2}$ are algebras, then

$$
s-\operatorname{deg}\left(A_{1} \oplus A_{2}\right)=\min \left\{s-\operatorname{deg}\left(A_{1}\right), s-\operatorname{deg}\left(A_{2}\right)\right\} .
$$

Lemma 2.3. If $A_{1}$ and $A_{2}$ are algebras, then

$$
s-\operatorname{deg}\left(A_{1} \otimes A_{2}\right) \geq \max \left\{s-\operatorname{deg}\left(A_{1}\right), s-\operatorname{deg}\left(A_{2}\right)\right\} .
$$


Proof. If the strong degree of $t_{1} \in A_{1}$ is $>n$, then the strong degree of $t_{1} \otimes 1 \in$ $A_{1} \otimes A_{2}$ is also $>n$. Using this, one easily completes the proof.

By a central simple algebra we mean a simple algebra such that its center coincides with $F$. The next lemma follows from [9, Lemma 2.3 and Corollary C.3].

Lemma 2.4. If $A$ is a central simple algebra, then $s-\operatorname{deg}(A)=\sqrt{\operatorname{dim} A}$.

Let us point out that the case when $A$ is infinite dimensional is not excluded here; in this case we have $s-\operatorname{deg}(A)=\infty$.

For other examples of algebras whose strong degree can be computed, we refer the reader to [5] and [9].

2.3. Lie (super)automorphisms. Let $B$ be an associative algebra. Recall that a bijective linear map $\Phi: B \rightarrow B$ is said to be a Lie automorphism if $\Phi([a, b])=$ $[\Phi(a), \Phi(b)]$ for all $a, b \in B$. Clearly, the restriction of a Lie superautomorphism of $A=A_{0} \oplus A_{1}$ on $A_{0}$ is a Lie automorphism of $A_{0}$. The next result is an immediate corollary to [9, Theorem 2.19 and Theorem 6.1]. Its proof is a typical application of the general theory of functional identities.

Theorem 2.5. Let $B$ be an associative algebra such that $s-\operatorname{deg}(B) \geq 3$. Assume that the center $Z$ of $B$ does not contain idempotents different from 0 and 1 . If $\Phi$ is a Lie automorphism of $B$, then $\Phi=\Theta+\Omega$, where $\Theta$ is either a homomorphism of $B$ or the negative of an antihomomorphism of $B$, and $\Omega$ is a map from $B$ into $Z$ which vanishes on commutators.

Note that this is the optimal description of a Lie automorphism through associative maps. Namely, a map of the form $\Theta+\Omega$, where $\Theta$ and $\Omega$ are as in the theorem, preserves the Lie bracket [., .]. It is easy to guess what are the counterparts of these maps in the superalgebra setting. Let $A=A_{0} \oplus A_{1}$ be an associative superalgebra. A linear map $\theta: A \rightarrow A$ is called a superhomomorphism if it is a homomorphism of the algebra $A$ (i.e., it satisfies $\theta(a b)=\theta(a) \theta(b))$ and if it preserves the $\mathbb{Z}_{2}$-grading (i.e., $\left.\theta\left(A_{i}\right) \subseteq A_{i}, i=0,1\right)$. Of course, superhomomorphisms also preserve the superbracket $[., .]_{s}$. Next, a linear $\mathbb{Z}_{2}$-grading-preserving map $\theta: A \rightarrow A$ is called a superantihomomorphism if $\theta(a b)=(-1)^{|a||b|} \theta(b) \theta(a)$ for all homogeneous elements $a, b \in A$. Note that the negative of a superantihomomorphism preserves the superbracket. Finally, if $\theta$ is either a superhomomorphism or a superantihomomorphism and $\tau$ is a map from $A$ into its center such that $\tau\left(\left[A_{0}, A_{0}\right]\right)=\tau\left(A_{1} \circ A_{1}\right)=\tau\left(A_{1}\right)=0$, then $\theta+\tau$ also preserves the superbracket (here by the center we mean the usual center, not supercenter). Moreover, if the range of $\tau$ lies in $A_{0}$, then $\theta+\tau$ also preserves the $\mathbb{Z}_{2}$-grading.

\section{MAIN RESULTS}

Let us first reveal the main idea on which this paper is based. Let $A=A_{0} \oplus A_{1}$ be an associative superalgebra, and let $\varphi: A \rightarrow A$ be a Lie superautomorphism. We "extend" $\varphi$ to $\Phi: G(A) \rightarrow G(A)$ in an obvious way, i.e., as the restriction of $\varphi \otimes$ id to $G(A)$. Thus

$$
\Phi\left(a_{i} \otimes g_{i}\right)=\phi\left(a_{i}\right) \otimes g_{i}, \quad a_{i} \in A_{i}, g_{i} \in G_{i}, i=0,1 .
$$


One easily checks that $\Phi$ is a Lie automorphism of $G(A)$. For example, if $a_{1}, b_{1} \in A_{1}$ and $g_{1}, g_{1}^{\prime} \in G_{1}$, then $g_{1} g_{1}^{\prime}+g_{1}^{\prime} g_{1}=0$ and hence

$$
\begin{aligned}
& \Phi\left(\left[a_{1} \otimes g_{1}, b_{1} \otimes g_{1}^{\prime}\right]\right)=\Phi\left(a_{1} \circ b_{1} \otimes g_{1} g_{1}^{\prime}\right)=\varphi\left(a_{1} \circ b_{1}\right) \otimes g_{1} g_{1}^{\prime} \\
= & \left(\varphi\left(a_{1}\right) \circ \varphi\left(b_{1}\right)\right) \otimes g_{1} g_{1}^{\prime}=\left[\varphi\left(a_{1}\right) \otimes g_{1}, \varphi\left(b_{1}\right) \otimes g_{1}^{\prime}\right]=\left[\Phi\left(a_{1} \otimes g_{1}\right), \Phi\left(b_{1} \otimes g_{1}^{\prime}\right)\right] .
\end{aligned}
$$

Similarly one considers the action of $\Phi$ on other commutators.

Now assume that the algebra $B=G(A)$ satisfies the conditions of Theorem 2.5. Then $\Phi=\Theta+\Omega$, where $\Theta$ and $\Omega$ are as in this theorem. We now have to use this information to describe $\varphi$. This is the idea of our proof.

Let us simplify our task by assuming slightly more than required in Theorem 2.5 , The assumptions that we impose are:

(a) $s-\operatorname{deg}\left(A_{0}\right) \geq 3$,

(b) the supercenter of $A$ is equal to $F$.

Note that (a) yields that $s-\operatorname{deg}\left(A_{0} \otimes G_{0}\right) \geq 3$ (by Lemma 2.3) and therefore $s-\operatorname{deg}(G(A)) \geq 3$ (by Lemma 2.11). Further, it is easy to see that (b) implies that the center $Z$ of $G(A)$ is equal to $1 \otimes G_{0}$. Since $G_{0}$ does not contain nontrivial idempotents, the same holds for $Z$. Therefore (a) and (b) indeed imply all assumptions of Theorem 2.5. We thus have $\Phi=\Theta+\Omega$. Note also that we can write $\Omega(r)=1 \otimes \omega(r), r \in G(A)$, where $\omega: G(A) \rightarrow G_{0}$. Finally we remark that (b) implies that elements from $F$ are the only elements that lie in both $A_{0}$ and the center of the algebra $A$.

We now have to treat two cases, the first, where $\Theta$ is a homomorphism and the second, where $\Theta$ is the negative of an antihomomorphism. Let us consider in detail the second case, which is apparently the less favorable.

We begin by considering $\varphi\left(a_{0} b_{0}\right) \otimes 1$ with $a_{0}, b_{0} \in A_{0}$. We have

$$
\begin{aligned}
& \varphi\left(a_{0} b_{0}\right) \otimes 1=\Phi\left(a_{0} b_{0} \otimes 1\right) \\
= & \Theta\left(\left(a_{0} \otimes 1\right)\left(b_{0} \otimes 1\right)\right)+1 \otimes \omega\left(a_{0} b_{0} \otimes 1\right) \\
= & -\Theta\left(b_{0} \otimes 1\right) \Theta\left(a_{0} \otimes 1\right)+1 \otimes \omega\left(a_{0} b_{0} \otimes 1\right) \\
= & -\left(\varphi\left(b_{0}\right) \otimes 1-1 \otimes \omega\left(b_{0} \otimes 1\right)\right)\left(\varphi\left(a_{0}\right) \otimes 1-1 \otimes \omega\left(a_{0} \otimes 1\right)\right)+1 \otimes \omega\left(a_{0} b_{0} \otimes 1\right) \\
= & -\varphi\left(b_{0}\right) \varphi\left(a_{0}\right) \otimes 1+\varphi\left(b_{0}\right) \otimes \omega\left(a_{0} \otimes 1\right)+\varphi\left(a_{0}\right) \otimes \omega\left(b_{0} \otimes 1\right) \\
& +1 \otimes\left(\omega\left(a_{0} b_{0} \otimes 1\right)-\omega\left(b_{0} \otimes 1\right) \omega\left(a_{0} \otimes 1\right)\right) .
\end{aligned}
$$

Thus,

$$
\begin{aligned}
\left(\varphi\left(a_{0} b_{0}\right)\right. & \left.+\varphi\left(b_{0}\right) \varphi\left(a_{0}\right)\right) \otimes 1=\varphi\left(b_{0}\right) \otimes \omega\left(a_{0} \otimes 1\right) \\
& +\varphi\left(a_{0}\right) \otimes \omega\left(b_{0} \otimes 1\right)+1 \otimes\left(\omega\left(a_{0} b_{0} \otimes 1\right)-\omega\left(b_{0} \otimes 1\right) \omega\left(a_{0} \otimes 1\right)\right) .
\end{aligned}
$$

For every $a_{0} \in A_{0}$ we write $\omega\left(a_{0} \otimes 1\right)=\tau\left(a_{0}\right)+\epsilon\left(a_{0}\right)$, where $\tau\left(a_{0}\right) \in F$ and $\epsilon\left(a_{0}\right)$ lies in the linear span of the products of $e_{i}$ 's. Suppose there exists $a_{0} \in A_{0}$ such that $\epsilon\left(a_{0}\right) \neq 0$. Then it readily follows from (2) that every $\varphi\left(b_{0}\right)$ lies in the linear span of $\varphi\left(a_{0}\right)$ and 1. But this is impossible since (a) in particular implies that $A_{0}$ contains elements that are not algebraic of degree $\leq 2$. Consequently $\epsilon\left(a_{0}\right)=0$ for every $a_{0} \in A_{0}$, and so $\omega\left(a_{0} \otimes 1\right)=\tau\left(a_{0}\right) \in F$. Therefore (2) reduces to

$$
\varphi\left(a_{0} b_{0}\right)+\varphi\left(b_{0}\right) \varphi\left(a_{0}\right)=\tau\left(a_{0}\right) \varphi\left(b_{0}\right)+\tau\left(b_{0}\right) \varphi\left(a_{0}\right)+\tau\left(a_{0} b_{0}\right)-\tau\left(b_{0}\right) \tau\left(a_{0}\right) .
$$

We now define $\theta: A_{0} \rightarrow A_{0}$ by

$$
\theta\left(a_{0}\right)=\varphi\left(a_{0}\right)-\tau\left(a_{0}\right),
$$


so that $\Theta\left(a_{0} \otimes 1\right)=\theta\left(a_{0}\right) \otimes 1$. Note that (3) can now be written as

$$
\theta\left(a_{0} b_{0}\right)=-\theta\left(b_{0}\right) \theta\left(a_{0}\right) \text {. }
$$

In a similar fashion we consider $\varphi\left(a_{0} b_{0}\right) \otimes e_{1} e_{2}$ with $a_{0}, b_{0} \in A_{0}$ :

$$
\begin{aligned}
& \varphi\left(a_{0} b_{0}\right) \otimes e_{1} e_{2}=\Phi\left(a_{0} b_{0} \otimes e_{1} e_{2}\right) \\
= & \Theta\left(\left(a_{0} \otimes 1\right)\left(b_{0} \otimes e_{1} e_{2}\right)\right)+1 \otimes \omega\left(a_{0} b_{0} \otimes e_{1} e_{2}\right) \\
= & -\Theta\left(b_{0} \otimes e_{1} e_{2}\right) \Theta\left(a_{0} \otimes 1\right)+1 \otimes \omega\left(a_{0} b_{0} \otimes e_{1} e_{2}\right) \\
= & -\left(\varphi\left(b_{0}\right) \otimes e_{1} e_{2}-1 \otimes \omega\left(b_{0} \otimes e_{1} e_{2}\right)\right)\left(\theta\left(a_{0}\right) \otimes 1\right)+1 \otimes \omega\left(a_{0} b_{0} \otimes e_{1} e_{2}\right) \\
= & -\varphi\left(b_{0}\right) \theta\left(a_{0}\right) \otimes e_{1} e_{2}+\theta\left(a_{0}\right) \otimes \omega\left(b_{0} \otimes e_{1} e_{2}\right)+1 \otimes \omega\left(a_{0} b_{0} \otimes e_{1} e_{2}\right) .
\end{aligned}
$$

Thus

$$
\left(\varphi\left(a_{0} b_{0}\right)+\varphi\left(b_{0}\right) \theta\left(a_{0}\right)\right) \otimes e_{1} e_{2}=\theta\left(a_{0}\right) \otimes \omega\left(b_{0} \otimes e_{1} e_{2}\right)+1 \otimes \omega\left(a_{0} b_{0} \otimes e_{1} e_{2}\right) .
$$

Using (44) we see that $\varphi\left(a_{0} b_{0}\right)+\varphi\left(b_{0}\right) \theta\left(a_{0}\right)=\tau\left(b_{0}\right) \theta\left(a_{0}\right)+\tau\left(a_{0} b_{0}\right)$, and so we get

$$
\theta\left(a_{0}\right) \otimes\left(\omega\left(b_{0} \otimes e_{1} e_{2}\right)-\tau\left(b_{0}\right) e_{1} e_{2}\right)+1 \otimes\left(\omega\left(a_{0} b_{0} \otimes e_{1} e_{2}\right)-\tau\left(a_{0} b_{0}\right) e_{1} e_{2}\right)=0 .
$$

Choosing $a_{0}$ so that $\theta\left(a_{0}\right) \notin F$ (its existence is a trivial consequence of (a)) it follows that for every $b_{0} \in A_{0}$ we have

$$
\omega\left(b_{0} \otimes e_{1} e_{2}\right)=\tau\left(b_{0}\right) e_{1} e_{2} .
$$

Next we consider $\varphi\left(a_{0} b_{1}\right) \otimes e_{1}$ with $a_{0} \in A_{0}, b_{1} \in A_{1}$. We have

$$
\begin{aligned}
& \varphi\left(a_{0} b_{1}\right) \otimes e_{1}=\Phi\left(a_{0} b_{1} \otimes e_{1}\right) \\
= & \Theta\left(\left(a_{0} \otimes 1\right)\left(b_{1} \otimes e_{1}\right)\right)+1 \otimes \omega\left(a_{0} b_{1} \otimes e_{1}\right) \\
= & -\Theta\left(b_{1} \otimes e_{1}\right) \Theta\left(a_{0} \otimes 1\right)+1 \otimes \omega\left(a_{0} b_{1} \otimes e_{1}\right) \\
= & -\left(\varphi\left(b_{1}\right) \otimes e_{1}-1 \otimes \omega\left(b_{1} \otimes e_{1}\right)\right)\left(\theta\left(a_{0}\right) \otimes 1\right)+1 \otimes \omega\left(a_{0} b_{1} \otimes e_{1}\right) \\
= & -\varphi\left(b_{1}\right) \theta\left(a_{0}\right) \otimes e_{1}+\theta\left(a_{0}\right) \otimes \omega\left(b_{1} \otimes e_{1}\right)+1 \otimes \omega\left(a_{0} b_{1} \otimes e_{1}\right),
\end{aligned}
$$

and hence

$$
\left(\varphi\left(a_{0} b_{1}\right)+\varphi\left(b_{1}\right) \theta\left(a_{0}\right)\right) \otimes e_{1}=\theta\left(a_{0}\right) \otimes \omega\left(b_{1} \otimes e_{1}\right)+1 \otimes \omega\left(a_{0} b_{1} \otimes e_{1}\right) .
$$

Since $\omega\left(b_{1} \otimes e_{1}\right), \omega\left(a_{0} b_{1} \otimes e_{1}\right) \in G_{0}$ it follows that $\varphi\left(a_{0} b_{1}\right)+\varphi\left(b_{1}\right) \theta\left(a_{0}\right)=0$. Consequently, choosing $a_{0}$ so that $\theta\left(a_{0}\right) \notin F$ we obtain $\omega\left(b_{1} \otimes e_{1}\right)=0$ for every $b_{1} \in A_{1}$.

We now extend $\theta$ to $A$ by setting

$$
\theta\left(a_{1}\right)=\varphi\left(a_{1}\right)
$$

for every $a_{1} \in A_{1}$. Note that we have

$$
\theta\left(a_{0} b_{1}\right)=-\theta\left(b_{1}\right) \theta\left(a_{0}\right)
$$

and $\Theta\left(b_{1} \otimes e_{1}\right)=\theta\left(b_{1}\right) \otimes e_{1}$. Of course, similarly we have $\Theta\left(b_{1} \otimes e_{i}\right)=\theta\left(b_{1}\right) \otimes e_{i}$ for every $i$.

Considering $\theta\left(b_{1} a_{0}\right) \otimes e_{1}=\varphi\left(b_{1} a_{0}\right) \otimes e_{1}$ in a similar (although now more straightforward) way as we considered $\varphi\left(a_{0} b_{1}\right) \otimes e_{1}$, one obtains

$$
\theta\left(b_{1} a_{0}\right)=-\theta\left(a_{0}\right) \theta\left(b_{1}\right)
$$

for all $a_{0} \in A_{0}, b_{1} \in A_{1}$. 
Finally we consider $\varphi\left(a_{1} b_{1}\right) \otimes e_{1} e_{2}$ with $a_{1}, b_{1} \in A_{1}$. Using (5) we obtain

$$
\begin{aligned}
& \varphi\left(a_{1} b_{1}\right) \otimes e_{1} e_{2}=\Phi\left(a_{1} b_{1} \otimes e_{1} e_{2}\right) \\
& =\Theta\left(\left(a_{1} \otimes e_{1}\right)\left(b_{1} \otimes e_{2}\right)\right)+1 \otimes \omega\left(a_{1} b_{1} \otimes e_{1} e_{2}\right) \\
& =-\Theta\left(b_{1} \otimes e_{2}\right) \Theta\left(a_{1} \otimes e_{1}\right)+1 \otimes \tau\left(a_{1} b_{1}\right) e_{1} e_{2} \\
& =-\left(\theta\left(b_{1}\right) \otimes e_{2}\right)\left(\theta\left(a_{1}\right) \otimes e_{1}\right)+1 \otimes \tau\left(a_{1} b_{1}\right) e_{1} e_{2} \\
& =\left(\theta\left(b_{1}\right) \theta\left(a_{1}\right)+\tau\left(a_{1} b_{1}\right)\right) \otimes e_{1} e_{2} .
\end{aligned}
$$

Therefore $\varphi\left(a_{1} b_{1}\right)=\theta\left(b_{1}\right) \theta\left(a_{1}\right)+\tau\left(a_{1} b_{1}\right)$, which yields

$$
\theta\left(a_{1} b_{1}\right)=\theta\left(b_{1}\right) \theta\left(a_{1}\right) \text {. }
$$

From (4), (6), (7) and (8) we now see that $\theta$ is the negative of a superantihomomorphism.

Extending $\tau$ to $A$ by simply setting $\tau\left(A_{1}\right)=0$ we thus have

$$
\varphi(a)=\theta(a)+\tau(a)
$$

for every $a \in A$. Let us finally make use of the condition that $\Omega$ vanishes on commutators (see Theorem 2.5). Considering commutators in $\left[A_{0} \otimes 1, A_{0} \otimes 1\right]$ we obtain $\tau\left(\left[A_{0}, A_{0}\right]\right)=0$. Similarly, considering commutators in $\left[A_{1} \otimes e_{1}, A_{1} \otimes e_{2}\right]$ and also applying (5) we get $\tau\left(A_{1} \circ A_{1}\right)=0$.

As $\theta$ is the negative of a superantihomomorphism, we have $\theta(1) \theta(a)=\theta(a) \theta(1)=$ $-\theta(a)$ for every $a \in A$. In particular, $\theta(1)$ thus commutes with all elements from $\varphi(A)=A$, which implies that $\theta(1) \in F$ (see the paragraph following (b)). But then $\theta(1)=-1$.

Suppose that $a=a_{0}+a_{1}$ is such that $\theta(a)=0$. Then $\varphi\left(a_{0}\right)+\tau\left(a_{0}\right)=0$ and $\varphi\left(a_{1}\right)=0$. The second identity yields $a_{1}=0$. The first identity implies that $\varphi\left(\left[a_{0}, A\right]\right)=\left[\varphi\left(a_{0}\right), \varphi(A)\right]=-\left[\tau\left(a_{0}\right), A\right]=0$. Thus $\left[a_{0}, A\right]=0$ and so $a=a_{0} \in F$. However, since $\theta(1)=-1$, this is possible only if $a=0$. This proves that $\theta$ is injective. From $\theta(a-\tau(a))=\theta(a)-\tau(a) \theta(1)=\theta(a)+\tau(a)=\varphi(a)$ we see that $\theta$ is also surjective. Thus $\theta$ is the negative of a superantiautomorphism.

Recall that we have derived all these conclusions under the assumption that $\Theta$ is the negative of an antihomomorphism. If $\Theta$ were a homomorphism, then following the same procedure we would arrive at analogous conclusions, such as $\theta$ is then a superautomorphism.

To summarize, we have the desired conclusion $\varphi=\theta+\tau$ under the assumption that the conditions (a) and (b) are fulfilled. More precisely, the following is true.

Theorem 3.1. Let $A=A_{0} \oplus A_{1}$ be an associative superalgebra such that its supercenter is $F$ and $s-\operatorname{deg}\left(A_{0}\right) \geq 3$. Then every Lie superautomorphism $\varphi$ of $A$ takes the form $\varphi=\theta+\tau$, where $\theta$ is either a superautomorphism of $A$ or the negative of a superantiautomorphism of $A$, and $\tau$ is a map from $A$ into $F$ satisfying $\tau\left(\left[A_{0}, A_{0}\right]\right)=\tau\left(A_{1} \circ A_{1}\right)=\tau\left(A_{1}\right)=0$.

In our final result we apply Theorem 3.1 to a more concrete situation.

Corollary 3.2. Let $A$ be a central simple associative algebra. Let e be an idempotent in $A$ and consider $A$ as an associative superalgebra with respect to (1). If $\operatorname{dim} e A e>4$ and $\operatorname{dim}(1-e) A(1-e)>4$, then every Lie superautomorphism $\varphi$ of $A$ is of the form $\varphi=\theta+\tau$ where $\theta$ is either a superautomorphism of $A$ or the negative of a superantiautomorphism of $A$, and $\tau$ is a map from $A$ into $F$ satisfying $\tau\left(\left[A_{0}, A_{0}\right]\right)=\tau\left(A_{1} \circ A_{1}\right)=\tau\left(A_{1}\right)=0$. 
Proof. Both algebras $e A e$ and $(1-e) A(1-e)$ are also central simple. The simplicity can be easily checked. Let us show that they are central. This is undoubtedly known, but we still give a short proof for completeness. We want to show that the center of $e A e$ is equal to $F e$. Let eae be a nonzero element in the center of $e A$. Since $A$ is simple, there exists $x_{i}, y_{i} \in A$ such that $\sum_{i} x_{i} e a e y_{i}=1$. For every $x \in A$ we then have $x e=\sum_{i} x_{i} e a e y_{i} x e$. Since eae commutes with ey $y_{i} x e$ this implies $x e=\sum_{i} x_{i} e y_{i} x e a e$. Thus $x e=b x e a e$ for every $x \in A$ where $b=\sum_{i} x_{i} e y_{i}$. Accordingly, ybxeae $=y x e=$ byxeae for all $x, y \in A$. That is, $[b, A]$ Aeae $=0$. Since $A$ is simple it follows that $[b, A]=0$, and hence, $A$ being central, we have $b=\lambda \in F$. Returning to $x e=b x e a e$ it now follows that $e a e=\lambda^{-1} e$, as claimed.

Lemma 2.4 implies that the strong degree of both $e A e$ and $(1-e) A(1-e)$ is $\geq 3$. But then the strong degree of $A_{0}$ is also $\geq 3$ by Lemma 2.2 .

Using the fact that the center of $e A e$ is $F e$, one can easily show that the supercenter of $A$ is just $F$. All conditions of Theorem 3.1 are thus fulfilled and the result follows.

For example, Corollary 3.2 is applicable to the algebra $M(p \mid q)$ as long as $p>2$ and $q>2$. It is easy to see that in this situation the identities satisfied by $\tau$ imply that $\tau$ is necessarily a scalar multiple of the supertrace, i.e. the map given by $\left[\begin{array}{ll}A & B \\ C & D\end{array}\right] \mapsto \operatorname{tr}(A)-\operatorname{tr}(D)$, where tr denotes the trace.

Corollary 3.2 shows both the power and the limitations of our approach based on the strong degree and functional identities. While it covers a rather large class of associative superalgebras (which are possibly infinite dimensional), it fails in some specific situations related to low dimensional algebras.

\section{REFERENCES}

[1] Yu. Bahturin, M. Brešar, Lie gradings on associative algebras, J. Algebra 321 (2009), 264-283. MR2469360

[2] Yu. Bahturin, M. Brešar, I. Shestakov, Jordan gradings on associative algebras, Algebr. Represent. Theory, to appear.

[3] Yu. Bahturin, D. Fischman, S. Montgomery, On the generalized Lie structure of associative algebras, Israel J. Math. 96 (1996), 27-48. MR1432725 (98d:16048)

[4] Yu. Bahturin, O. H. Kegel, Universal sums of abelian subalgebras, Comm. Algebra 23 (1995), 2975-2990. MR.1332159 (96g:16033)

[5] K. I. Beidar, M. Brešar, M. A. Chebotar, Functional identities revised: The fractional and the strong degree, Comm. Algebra 30 (2002), 935-969. MR.1883042 (2002k:16048)

[6] K. I. Beidar, M. Brešar, M. A. Chebotar, Jordan superhomomorphisms, Comm. Algebra 31 (2003), 633-644. MR1968917 (2004a:16060)

[7] G. Benkart, X. Xu, K. Zhao, Classical Lie superalgebras over simple associative algebras, Proc. London Math. Soc. (3) 92 (2006), 581-600. MR2223537 (2007c:17006)

[8] M. Brešar, Commuting traces of biadditive mappings, commutativity-preserving mappings and Lie mappings, Trans. Amer. Math. Soc. 335 (1993), 525-546. MR1069746 (93d:16044)

[9] M. Brešar, M. A. Chebotar, W.S. Martindale III, Functional Identities, Birkhäuser Verlag, 2007. MR2332350 (2008j:16068)

[10] C. Gómez-Ambrosi, J. Laliena, I. P. Shestakov, On the Lie structure of the skew elements of a prime superalgebra with superinvolution, Comm. Algebra 28 (2000), 3277-3291. MR 1765316 (2001g:17007)

[11] C. Gómez-Ambrosi, I. P. Shestakov, On the Lie structure of the skew elements of a simple superalgebra with superinvolution, J. Algebra 208 (1998), 43-71. MR.1643975 (99j:17032)

[12] I. N. Herstein, Topics in Ring Theory, University of Chicago Press, 1969. MR0271135 $(42: 6018)$

[13] J. Laliena, S. Sacristán, Lie structure in semiprime superalgebras with superinvolution, J. Algebra 315 (2007), 751-760. MR.2351892(2008j:16123) 
[14] W. S. Martindale III, Lie isomorphisms of prime rings, Trans. Amer. Math. Soc. 142 (1969), 437-455. MR0251077 (40:4308)

[15] F. Montaner, On the Lie structure of associative superalgebras, Comm. Algebra 26 (1998), 2337-2349. MR 1626634 (99g:16055)

[16] S. Montgomery, Constructing simple Lie superalgebras from associative graded algebras, J. Algebra 195 (1997), 558-579. MR1469640 (99g:17043)

[17] Y. Wang, Functional identities and Lie superhomomorphisms on prime superalgebras, Comm. Algebra, to appear.

[18] K. Zhao, Simple Lie color algebras from graded associative algebras, J. Algebra 269 (2003), 439-455. MR2015286 (2004m:17044)

Department of Mathematics and Statistics, Memorial University of Newfoundland, St. John's, Newfoundland, A1C5S7, Canada

E-mail address: yuri@math.mun.ca

Faculty of Mathematics and Physics, University of Ljubljana, Jadranska 19, Ljubljana, Slovenia - and - Faculty of Natural Sciences and Mathematics, University of MARIBOR, KoroŠKa 160, Maribor, Slovenia

E-mail address: matej.bresar@fmf.uni-lj.si 